\title{
Ueber den Einfluss des Amylnitrits auf Gefässtonus und Herzschlag.
}

\author{
Von
}

\section{Dr. Wilhelm Filehme,}

Docenten an der Universität und erstem Assistenten an der medicinischen Poliklinik zu Erlangen.

Die Wirkung des salpetrigsauren Amyloxyds hatte ich in allerneuster Zeit bei einzelnen krankhaften Zuständen benutzt, um zu entscheiden, ob diesen eine abnorme Innervation der Hirngefässe zu Grunde liege oder nicht. Hierdurch war ich zu einer genaueren Durchsicht des literarischen Materials, welches über jenes Mittel vorliegt, geführt worden und habe mich veranlasst gesehen, einige auf dasselbe beziigliche Fragen zu bearbeiten.

Bekanntlich verursacht die Inhalation sehr geringer Mengen von Amylnitritdämpfen beim Menschen, wie Guthrie ${ }^{1}$ ) zuerst an sich selbst beobachtete, eine Röthung des Gesichts und eine Beschleunigung des Herzschlages bis zum Doppelten der normalen Schlagzahl.

Die physiologische Grundlage dieser beiden Erscheinungen ist meiner Meinung nach bisher noch nicht genügend erforscht; ich habe deshalb auf diese Puncte eine Untersuchung gerichtet, welche ich im hiesigen physiologischen Institute ausgeführt habe und bei welcher mich Herr Professor Rosenthal durch Rath und That in freundlichster Weise unterstützt hat; auch will ich nicht unterlassen der Liebenswürdigkeit zu gedenken, mit welcher College Wolffberg mir bei meinen Versuchen stets zur Seitc gestanden hat.

\section{Die Wirkung des Amylnitrits anf den Gefässtonus.}

Die Röthung des Gesichts, welche nach Inhalation von Amylnitrit in so frappanter Weise auftritt, hätte entweder von einer Vermehrung der Blutzufuhr oder einer Behinderung der Blutausfuhr

1) The Quarterly Journal of the Chemical Society of London. Vol. XI. 1859. p. 245. 
abhängig sein können, und für jeden dieser beiden Vorgänge wären verschiedene Möglichkeiten der Entstehung gewesen. Die sphygmographischen und namentlich die manometrischen Untersuchungen Gamgee's ${ }^{1}$ ) hatten nun gezeigt, dass unter dem Einflusse des Amylnitrits bei Mensch und Säugethier die Spannung im Arteriensysteme abnimmt. Da die Herzthätigkeit während der Einwirkung des Mittels frequenter als in der Norm ist und eher verstärkt als abgeschwächt erscheint, so musste aus Gamgee's Beobachtungen der Schluss gezogen werden, dass die Abnahme der Spannung des Arteriensystems auf einer Verminderung der peripherischen Widerstände, d. h. auf einer Erweiterung der kleinsten peripherischen Arterien beruhe: auf diesen Vorgang war dann das Erröthen leicht zurückzuführen; die Füllung der Capillaren des Gesichtes musste jetzt um so eher eine maximale werden, als das Herz, wie Rich a rd$\operatorname{son}^{2}$ ) betont, häufiger und kräftiger sich contrahirend, grössere Blutmengen in das erweiterte Strombett überführt.

Es entstand nun die Frage, welches die nähere Ursache der Arterienerweiterung sei.

Während Richardson ${ }^{3}$ ) ohne experimentellen Beweis die Vermuthung aussprach, dass das Amylnitrit auf die vasomotorischen Nerven lähmend wirke, stellte sich Lauder Brunton ${ }^{4}$ ) die Aufgabe, experimentell $\mathrm{zu}$ entscheiden, ob die nach Inhalation von Amylnitritdämpfen entstehende Erweiterung der Arterien von einer Lähmung des vasomotorischen Centrums oder von einer Lähmung der Gefässe selber abhängig sei. Ausgehend von der bis vor Kurzem noch ziemlich allgemein verbreiteten Ansicht, dass das Centrum für die Vasomotion ausschliesslich in der Medulla oblongata gelegen sei, glaubte Lauder Brunton diese Frage dadurch erledigen zu können, dass er die Wirkung des Amylnitrits auf den Blutdruck bei Kaninchen untersuchte, denen er das Halsmark durchschnitten hatte; denn jetzt wähnte er die Gefässe von jeder centralen Innervation befreit. Da auch in diesen Versuchen der durch die Marktrennung bereits sehr erniedrigte Blutdruck eine weitere Herabminderung durch die Amylnitriteinblasungen erfuhr, so war

1) Persönl. Mittheilung an Lauder Brunton: Berichte üb. d. Verh. d. Kön. Sächs. Ges. d. W. zu Leipz. 1869. p. 285 u. 286.

2) The Medical Times and Gaz. 1863. Sept. 26. p. 334.

3) 1. c. p. 335 u. ebenda 1870. II p. 472.

4) Berichte üb. d. Verh. d. K. S. Ges. d. W. zu Leipz. 1869. p. 285 ff. 
Lauder Brunton von seinem Standpuncte aus zu dem Schlusse gezwungen, dass jenes Mittel die ihres Centrums beraubten Gefässe direct gelähmt habe, und er musste daher annehmen, dass die gefässerweiternde Wirkung des Amylnitrits auf der Fähigkeit dieses Mittels beruhe, die Gefässe selber und nicht ihr Centrum zu lähmen.

Indessen können wir nach unserem heutigen Wissen die Richtigkeit der Lauder Brunton'schen Voraussetzung und also auch die Gültigkeit seiner Schlussfolgerung nicht anerkennen. Die Ar-beiten von $\mathrm{Goltz}^{1}$ ), Schlesing $\mathrm{r}^{2}$ ) und Vulpia $\mathrm{n}^{3}$ ) beweisen, dass die Körpergefässe eines Thieres, dem das Halsmark durchschnitten ist, keineswegs als nicht innervirt angesehen werden dürfen, indem auch das Rückenmark vasomotorische Centralapparate enthält. Und deshalb ist durch die im Uebrigen sehr interessanten und sorgfältigen Untersuchungen Lauder Brunton's die vorliegende Frage durchaus nicht beantwortet.

Bernheim ${ }^{4}$ ) hat auf Bernstein's Veranlassung diese Angelegenheit noch einmal in Untersuchung gezogen. Er zeigt, dass die durch Amylnitrit erweiterten Ohr- und Eingeweide-Arterien eines Kaninchens durch elektrische Reizung der zugehörigen vasomotorischen Nervenstämme (Sympathicus, Splanchnicus) verengert werden, was seiner Meinung nach nicht der Fall sein könnte, wenn die Gefässe selber gelähmt wären. Aus seiner Beobachtung folgert Bernheim demgemäss, dass nicht die Arterien und nicht die vasomotorischen Nervenstämme, sondern die vasomotorischen Centralapparate durch das Amylnitrit gelähmt werden.

Diese Auffassung ist von $P i \mathbf{k}^{5}$ ) angegriffen worden: Bernheim's Versuch beweise nur, dass der elektrische Reiz, welcher den Nerven traf, stärker war als die erschlaffende Wirkung des Amylnitrits auf die Gefässmusculatur; letztere dürfe nämlich nicht

1) Virch. Arch. Bd. 28 p. 428 ; Bd. 29 p. 294 . Centralbl. f. d. m. W. 1864 Nr. 40. Pflüger's Arch. Bd. VIII (1873) (p. 460-498) p. 482-495. Von der n eu es t e $n$ Veröffentlichung Gol t $z$ 's: über sgefässerweiternde Nerven “ sehe ich $a b$, da sie durch die auf Goltz's Veranlassung unternommene Untersuchung von Putzeys und Tarchan off (Centrabl. f. d. m. W. 1874. Nr. 40) bereits widerlegt ist.

2) Wiener med. Jahrb. 1874. I p. 1-29. ,

3) Compt. rend. 1874 LXXVIII n. 7 p. $472-476$.

4) Pflüger's Arch. (1873) Bd. VIII p. 253.

5) จUeb. d. Amylnitrit u: s. therap. Anw. Inaug.-Diss. Bonn 1874. 
Ueber den Einfluss des Amylnitrits auf Gefässtonus und Herzschlag. 473

als todt, als vollständig gelähmt, sondern nur als erschlafft angesehen werden.

In der That ist dieser Einwand Pick's durchaus berechtigt: damit eine Gefässdilatation der kleineren Arterien entstehe, genügt es, dass, bei gleichbleibender vasomotorischer Innervation, die Erregbarkeit der Gefässmusculatur um einen gewissen Theil ihres ursprïnglichen Werthes sinke; cine elektrische Reizung des zugehörigen Nerven wird dann bei einer gewissen Stärke eine Verengerung der Arterien herbeiführen und doch war in solchem Falle die Ursache der Erweiterung in der Gefässmusculatur und nicht in den Centren gelegen.

Nach Entkräftung der Bernheim'schen Schlussfolgerung sieht sich Pick veranlasst, Lauder Brunton's Auffassung aufrecht zu erhalten und den Grund der Gefässerweiteruug in den Gefässen selber zu suchen. Er hält nicht nur die (oben von mir widerlegte) Beweisführung Lauder Brunton's für zutreffend, sondern findet, wie bereits vor ihm Wood'), eine Stütze für die von ihm vertretene Anschauung besonders in dem Umstande, dass sich das Amylnitrit als ein directes Muskelgift erwiesen hat.

Es liegt aber auf der Hand, dass mit der Hinfälligkeit der Lauder Brunton'schen Beweisführung diese letztere von Pick angeführte Erfahrung nicht als beweisendes Argument angesehen werden kann. Ausserdem aber hat sich ihm das Amylnitrit als Muskelgift nur bei massenhafter directer Application (flüssig und gasförmig) und noch dazu auf quergestreifte Muskelfasern und auf Infusorien gezeigt; dagegen hat Pick nicht nachgewiesen, dass auch die in der Gefässwand vorhandenen glatten Muskelfasern nach Inhalationen sei es selbst tödtlicher Mengen unserer Substanz ihre Lebenseigenschaften verlieren.

Somit hat auch die Arbeit Pick's für die exacte Beantwortung der uns beschäftigenden Frage kein positives Material gebracht.

Schüller ${ }^{2}$ ) vermeint neue Beweise dafür geliefert zu haben, dass, wie Lauder Brunton und Pick es annehmen, die Gefässerweiterung bei Einwirkung von Amylnitrit auf einer directen Er-

1) Americ. Journal of the Med. Scienc. 1871. (Vol. LXII resp. CXXIII) (p. 39-65 und ebenda p. 359 ff.) p. 53.

2) Berl. klin. Wochenschr. 1874. Nr. 25. 
schlaffung der Gefässmusculatur beruhe: er durchschnitt an Kaninchen den Sympathicus; nachdem sich die in Folge dieser Operation bekanntlich entstehende Erweiterung der Ohrgefässe ausgebildet hatte, setzte Schüller die Thiere der Einwirkung von Amylnitrit aus und beobachtete dann eine neue Erweiterung. Nun glaubt Schüller, dass die Durchtrennung des Sympathicus die Gefässe von jeder centralen Innervation abgeschnitten habe, und muss daher die neue Erweiterung auf eine directe Lähmung der Gefàsse beziehen. Dieser Schluss würde richtig sein; die Voraussetzung ist aber unerwiesen und wie wir sogleich sehen werden sogar unrichtig. Es treten zum Kaninchenohre ausser den vom Sympathicus kommenden verschiedene Nervenzweige, nämlich vom Vagus, vom Plexus cervicalis, vom Quintus und vom Facialis, und Schüller hätte erst den Beweis zu bringen, dass in jenen Nervenbahnen keine vasomotorischen Fasern verlaufen. Nun wissen wir aber sogar durch Schiff'), dass beim Kaninchen im Ramus auricularis cervicalis stets vasomotorische Fasern enthalten sind, ja dass zuweilen solche Fasern ausschliesslich oder zum grössesten Theile in diesem Nerven sich befinden, so dass dann der Sympathicus deren gar keine oder nur sehr wenige birgt. Auch Brown-Séquard und Valent in haben zugegeben, dass zuweilen die vasomotorischen Fasern ausserhalb des Sympathicus verlaufen. Ich habe erst kürzlich ebenfalls bei zwei Kaninchen nach Durchschneidung des Sympathicus keine Dilatation der Ohrgefässe erhalten, während dic Pupille eng wurde. Ferner hat Claude-Bernard gefunden, dass die Durchtrennung des Facialis nach seinem Austritte aus dem Foramen stylomastoideum die Temperatur des Kaninchenohres derselben Seite erhöht, und Sch iff hat bewiesen, dass dies von vasomotorischen Fasern herrührt, welche der genannte Nerv vom Vagus erhält. Die Voraussetzungen Schüller's sind also unrichtig und daher ist seine Schlussfolgerung unzulässig.

Ueberblicken wir jetzt die vorliegenden Thatsachen, so steht allein fest, dass das Amylnitrit die Arterien erweitert; unentschieden ist dagegen, welcher Ort des vasomotorischen Gesammtapparates der Angriffspunct für jene Substanz ist.

Bei dem Mangel an beweisenden Thatsachen versuchte ich es, mir aus der Betrachtung der Amylnitritwirkung am Menschen eine

1) Unters. z. Phys. d. Nervensyst. etc. Frankf. 1855. Bd. I p. 155 ff. 
Vermuthung darüber abzuleiten, wie jene Wirkung zu Stande kommen möchte.

Die Röthe, welche beim Menschen nach Amylnitrit-Inhalation auftritt, erstreckt sich über dieselben Hautabschnitte, über welche sich die Schamröthe zu verbreiten pflegt, nämlich über das Gesicht, den Hals und den obersten Theil der Brust.' In der Mitte der Brust, entsprechend dem Sternum, geht sie meist etwas tiefer nach abwärts als an den Seiten. Auch Darwin ${ }^{1}$ ) erwähnt bei der Besprechung des durch Gemüthsbewegungen herbeigeführten Erröthens das Rothwerden nach Amylnitrit-Inhalation und sagt: „Dieses Rothwerden ist dem Erröthen vor Scham fast in jeder Einzelheit ähnlich." Nach meiner Erfahrung ist die Amylnitrit-Röthe meistens eine ziemlich scharf abgegrenzte; hierin bin ich im Widerspruche zu Pick, welcher angibt, die Röthung nehme ganz allmälig nach der. untern Körperhälfte zu ab; indessen mögen hier eben solche indivividuelle Verschiedenheiten vorkommen, wie sie in Bezug auf die Ausdehnung der Schamröthe bestehen. Jedenfalls aber gibt es Fälle, in denen die Amylnitrit-Röthe verbältnissmässig scharf abgegrenzt ist; eine solche Erscheinung ist kaum vereinbar mit einer directen Lähmung der Gefässe selber; denn warum sollte von zwei an der Grenze liegenden Gefässen das eine gelähmt werden und das andere unversehrt bleiben, während in beiden doch Blut von gleicher Qualität fliesst? Dagegen wäre es viel leichter zu verstehen, dass jener nervöse Centralmechanismús, welcher die Arterien der genannten Partie innervirt und welcher in Momenten des Schamerröthens so prompt und fein auf die leichtesten ihm von den Organen der Seele zukommenden Erregungen seine Thätigkeit einstellt, auch durch das Amylnitrit, direct oder indirect, ausser Function gesetzt werde, und hierdurch die Gefässe sich erweitern lasse.

Jedoch waren exacte auf diesen Punct gerichtete Versuche nothwendig, um die Richtigkeit oder das Irrthümliche dieser Vorstellung festzustellen.

Meine Versuche haben nun ergeben, dass in der That der nervöse vasomotorische Centralapparat durch Inhalation geringer Mengen von Amylnitritdämpfen ausser Thätigkeit gesetzt wird,

1) Ier Ausdruck der Gemüthsbewegungen etc. Uebers. v. Carus. Stuttgart 1872. p. 331 u. 332. 
während die Gefässe und die vasomotorischen Nervenstämme intact bleiben.

Ich experimentirte mit einem von Herrn Professor $\mathrm{Hilger}$ hierselbst bereiteten Präparate, welches der genannte Herr in freigebigster Weise mir zu überlassen die Güte hatte. Die Mehrzahl der Versuche wurde an Kaninchen angestellt.

Die Methode, nach welcher ich die Kaninchen in allen Versuchen das Amylnitrit inhaliren liess, verdient eine nähere Besprechung. Ich machte stets vor dem Versuche die Tracheotomie und führte in das untere Ende der Trachea eine Canüle ein, durch welche die Thiere frei athmeten. Ein mit Amylnitrit getränkter, am Boden eines Becherglases befindlicher Wattebausch wurde während etwa drei Athemzügen in eine Entfernung von $1 / 2 \mathrm{Ctm}$. vor die Oeffnung der Canüle gebracht. Alsbald zeigt sich eine maximale Füllung der Ohrgefässe. Die Athemzüge, welche das Amylnitrit aufnehmen, werden von dem Kaninchen ohne jede Abweichung von der Norm ausgeführt.

Schon Lauder Brunton hat auf die Unzuträglichkeiten hingewiesen,' welche sich herausbilden, wenn man die 'Thiere durch die Nase inhaliren lässt. Sie halten alsdann sofort mit der Athmung inne und erst die allerhöchste Athemnoth, die bereits $\mathrm{Er}$ stickungserscheinungen hervorruft, zwingt die Kaninchen schliesslich die Amylnitritdämpfe in die Lunge zu ziehen. Ausserdem wissen wir aber durch die Untersuchungen von $\mathrm{Hol} \mathrm{mg} \mathrm{ren}^{1}$ ) und Kratschme ${ }^{2}$ ), dass bei Inhalation verschiedener Aether $u$. s. w. mehrfache Reflexe von den Trigeminusausbreitungen in der Nasenschleimhaut nicht nur auf die Athmung, sondern auch auf die Centren der Vasomotion und der Herzhemmung veranlasst werden, welche für die vorliegende Untersuchung sehr störend gewesen sein würden. Lauder Brunton vermied diese Störung dadurch, dass er die Thiere künstlich respirirte und ihnen die Dämpfe einbliess. Meine Methode ist offenbar besser. Zunächst wäre die künstliche Athmung in meinen Versuchen, ebenso wie sie es in denen Brunto n's war, eine überflüssige Complication gewesen, die, wo es geht, vermieden werden muss, weil ein künstlich ventilirtes Thier kein

1) Upsala Läk. Sällsk. Handl. (1867) Bd. II Nr. 3 p. 134.

2) Sitzungsber. d. math.-naturw, Cl, d. Kais. Ak, d. W. (Wien) 62. Bd. 2. Abth. 16. Sitzung (1870). 
Ueber den Einfluss des Amylnitrits auf Gefässtonus und Herzschlag. 477

normales ist: die künstliche Respiration erniedrigt ja den Blutdruck an und für sich und verändert den Gaszustand des Blutes. In Brunton's wie in meinen Versuchen war keine Veranlassung zur künstlichen Respiration als solcher; es handelte sich nur darum, die Amylnitritdämpfe in die Lungen des Thieres zu bringen und dies besorgen tracheotomirte Thiere allein und ohne Weiteres. Ferner war ich bei meiner Methode im Stande, die Zufuhr von Amylnitritdämpfen viel feiner zu reguliren als es Brunton vermochte. Dass Brunton mit viel zu grossen Gaben gearbeitet hat, geht aus seiner Angabe hervor, er sei gezwungen gewesen die Kaninchen zu curaresiren, weil sich alsbald nach Beginn der Einblasung allgemeine Krämpfe gezeigt hätten. Wenn man wie ich verfährt, kann man die Thiere bei enorm erweiterten Ohrgefässen beliebig lange Zeit völlig frei von Krämpfen halten. Eine feinere Regulirung der Amylnitritzufuhr ist dazu aber nothwendig: die Kaninchen sind eben ungemein empfindlich gegen unser Mittel.

Die maximale Erweiterung der Ohrgefässe nach Inhalation geringer Mengen Amylnitrits ist eine so constante, ihr Eintritt ein so plötzlicher, und ihre Dauer ist durch vorsichtiges ferneres Darreichen so beliebig zu verlängern, dass der Verdacht zurückgewiesen werden muss, als handelte es sich hierbei um eine Verwechslung mit dem normalen Gefässspiele des Kaninchenohres. Uebrigens geben sowohl Amez-Drozl) als auch Pick, Bernheim und Schüller die gleiche Beobachtung an, und es ist nicht zu verstehen, warum Eulenburg und Guttmann ${ }^{2}$ ) sagen: „Unsere Untersuchungen an den Ohrgefässen der Kaninchen - - liessen es unentschieden, ob dieselben nach Einwirkung des Amylnitrits wirklich erweitert werden." Diese Autoren haben einen Theil ihrer Versuche ebenfalls mit Inhalationen gemacht, bei denen sie 5-10 Tropfen mittels des Skinner'schen Apparates einathmen liessen, und haben nur eine "vorübergehende Obnubilation des Sensoriums" beobachtet. Wenn die Thiere bei der von diesen Autoren geübten Methode wirklich Amylnitrit in die Lunge bekommen haben sollten, so wüsste ich keine Erklärung, wieso die enorme Blutüberfüllung der Ohrgefässe von Eulenburg und Guttmann nicht gesehen worden ist. Da

1) Arch. de phys. norm. et pathol. 1873. p. 467-503.

2) Reichert's und du Bois-Reymond's Arch. 1873 (p. 442-448) p. 447 Anm. 1 . 
ihre Kaninchen jedoch $\mathrm{d} u \mathrm{rch}$ die Nase inhalirten, und da dieser Umstand, wie ich oben auseinandergesetzt habe, eine wesentliche Fehlerquelle mit sich bringt, da sie ferner nicht die Zeitdauer der Inhalation angeben, so steht zu vermuthen, dass ihre Versuchsthiere das Amylnitrit nicht in die Lunge gebracht haben. Diese Vermuthung wurde aber für mich zur Gewissheit, als ich den Einfluss untersuchte, den unser Mittel auf den Herzschlag und die Respiration ausuibt. Eulenburg und Guttmann geben an, dass in ihren Versuchen Respiration und Herzaction intact blieben. Ich werde später zeigen, dass diese beiden Functionen beim Kaninchen äusserst erhebliche Abweichungen zeigen, sobald geringe Mengen Amylnitrits eingeathmet werden. Um das so äusserst präcis wirkende Amylnitrit nicht in den Verdacht kommen zu lassen, als wäre seine Wirkung eine schwächliche und beim Kaninchen eine andere als beim Menschen, sehe ich mich daher zu der Behauptung genöthigt, dass die Kaninchen Eulenburg's und Guttmann's bei den Inhalationsversuchen überhaupt kein Amylnitrit in sich aufgenommen haben, und halte es der Unentschiedenheit dieser beiden Autoren gegenüber für erwiesen, dass die Ohrgefässe des Kaninchens sich nach Amylnitrit-Inhalation constant in maximalstem Maasse erweitern.

Bei meiner Untersuchung über die Angriffsweise des Amylnitrits ging ich zunächst von folgendem Gedanken aus:

Wenn das eingeathmete Amylnitrit in der That die Gefässe selber, also direct lähmt, so müsste diese Wirkung sich ganz besonders auf die Gefässe der Lunge erstrecken, weil an dieser Stelle das Blut mehr unzersetzten Amylnitrits enthalten würde als in den andern Körpercapillaren. Da nämlich die Amylnitritwirkung so sehr schnell vorübergeht, so muss angenommen werden, dass unser Mittel sehr schnell im Blute zersetzt oder eliminirt wird.

Um die Lungen dem Auge zugänglich zu machen, legte ich ein Thorax-Fenster von der Höhe eines Intercostalraumes und in der Länge von etwa $1 \mathrm{Ctm}$. in der Weise an, dass das parietale Blatt der Pleura frei zu Tage lag. Bei der Zartheit und Durchsichtigkeit der Kaninchenpleura kann man so die Gefässfüllung der Lungen sehr gut beurtheilen und ein Erröthen derselben künnte dem Beobachter nicht entgehen.

In den auf diese Weise angestellten Versuchen habe ich nach Inhalation von Amylnitritdämpfen kein Erröthen gesehen; und wenn 
Ueber den Einfluss des Amylnitrits auf Gefässtonus und Herzschlag. 479

die Ohren schon längst mit Blut überfüllt waren, zeigte die Lunge ihre normale Farbe. Aus dieser Beobachtung schliesse ich, dass die der Dampfaufnahme fähigen Gefässe der Lunge und die stromabwärts von ihnen gelegenen Blutgefässe von dem Amylnitrit nicht direct erweitert werden; es werden unmittelbar also bestimmt nicht erweitert: die Venen und die Capillaren und diejenigen feinsten Arteriolen, die noch etwa dem Blute einen Gasaustausch durch die Gefässwandung hindurch gestatten. Dagegen war mein Versuch nicht beweisend für die Frage, ob die mit stärkerer Musculatur versehenen Arterienzweige, welche stromaufwärts von der Zufuhrstelle lagen und zu welchen das mit Amylnitrit geschwängerte Blut erst nach Zurücklegung des ganzen Körperkreislaufs gelangte, nicht vielleicht doch direct durch das Amylnitrit gelähmt werden würden, wenn es sofort zu ihnen gelangt wäre.

Um die vollständige Entscheidung unserer Frage zu erzielen, wurde folgender Plan gefasst. Die Bahn, auf welcher die vasomotorische Erregung verläuft, sollte an einer Stelle abgebrochen werden, und es sollte die sonst vom vasomotorischen Centrum kommende Erregung durch einen Reiz ersetzt werden, welcher genau dasselbe leistete, was vorher die centrale Innervation geleistet hatte. Wenn dann Amylnitrit inhalirt wurde, so musste offenbar der Effect verschieden sein, je nachdem das Mittel direct erschlaffend auf die Gefässe wirkte oder sie ebenso wie die vasomotorischen Nervenstämme intact liess.

$\mathrm{Zu}$ diesem Zwecke wurde einem (tracheotomirten) Kaninchen der Sympathicus der einen (rechten) Seite durchschnitten. Nachdem sich die gewöhnliche, anscheinend maximale Erweiterung der Ohrgefässe ausgebildet hatte, reizte ich das peripherische Ende des Nerven mit Inductionsströmen, die ich von unwirksamen Stromstärken ausgehend so lange steigerte, bis eine mittlere Contraction der Gefässe des rechten Ohres wieder erreicht war, so dass der Füllungszustand der Ohrgefässe auf der rechten Seite dem mittleren Füllungszustande der linken, normal innervirten Ohrgefässe gleich war. Sobald das Kaninchen in der oben besprochenen Weise der Einwirkung von Amylnitritdämpfen ausgesetzt wurde, erweiterten sich die Gefässe des linken Ohres (deren Sympathicus intact war) alsbald ad maximum, während die Gefässe der verletzten Seite in mittlerer Contiaction verharrten. Hierin trat keine Aenderung 
ein, selbst wenn ich das Amylnitrit bis zum Eintritt allgemeiner Krämpfe darreichte.

Dieser Versuch gibt die endgiltige Entscheidung der uns beschäftigenden Frage.

Da in dem peripherischen Stücke des durchschnittenen rechten Sympathicus zu den Gefässen eine Erregung hinabfloss, welche genau so stark war, wie die, welche ihm in der Norm von dem vasomotorischen Centrum übertragen wird, und da im Uebrigen sich die Ohrgefässe der réchten und der linken Seite unter ganz gleichen Bedingungen befanden, so kann die Verschiedenheit ihres Verhaltens nur darauf bezogen werden, dass im linken (unverletzten) Sympathicus in Folge der Amylnitritwirkung die Erregung aufgehört hatte oder erheblich abgeschwächt war. Die Reaction des rechten (durchschnittenen) Nerven gegen den ausgeprobten elektrischen Reiz beweist, dass dieses Erlöschen der Erregung im linken (unverletzten) Nerven nicht auf eine Vernichtung seiner Erregbarkeit zurückgeführt werden darf; dieses Erlöschen muss vielmehr dadurch zu Stande gekommen sein, dass diesem in normaler Weise erregbar gebliebenen Nerven vom Centrum her keine Erregungen mehr übertragen wurden. Der Versuch beweist also, dass die Blutgefässe während der Amylnitritwirkung ihren normalen Tonus beibehalten, sobald ihnen auf der Bahn der vasomotorischen Nerven die normalen Erregungen zufliessen, und dass ferner während der Amylnitritintoxication die vasomotorischen Nerven von ihrer Erregbarkeit Nichts eingebüsst haben. Weder die Gefässe noch die vasomotorischen Nervenfasern werden also durch das Amylnitrit gelähmt.

Bei unserm heutigen Wissen wäre es voreilig, aus diesen Ergebnissen sofort den Schluss zu ziehen, dass das Amylnitrit die vasomotorischen Centralapparate lähme. Dieser Schluss wäre nur dann zulässig, wenn wir am Warmblüter keine anderen Ursachen einer Gefässdilatation kennten, als eine Lähmung entweder der Gefässmusculatur, oder der vasomotorischen Nervenfasern und ihrer Endigungen, oder des Centrums selber. Nun wissen wir aber durch die schöne Untersuchung von Ludwig und Cyon, dass man eine auf Erschlaffung kleinster Arterien beruhende Blutdrucksverminderung veranlassen kann, wenn man den von ihnen sogenannten Nervus depressor reizt. Bis jetzt kennt man nun in dem Körper der Säugethiere keinen anderen Nerven, der die gleiche Function 
Ueber den Einfluss des Amylnitrits auf Gefässtonus und Herzschlag. 481 hätte. Ich musste daher zunächst ermitteln, ob nicht vielleicht das Amylnitrit, indem es mit dem Blute zum Herzen gelangt, die auf dessen Innenfläche sich ausbreitenden Depressor-Endigungen erregt und auf diesem Wege die Blutdruckerniedrigung hervorbringt.

Der Versuch hat mich belehrt, dass dies nicht der Fall ist. Auch bei einem Kaninchen, dem beide Depressores durchschnitten sind, tritt nach Amylnitrit-Inhalation alsbald die enorme Dilatation der Ohrgefässe ein.

Somit müssen wir bei dem heutigen Stande unseres Wissens die durch Amylnitrit herbeigeführte Gefässdilatation auf eine Lähmung (Herabsetzung der Leistung) der vasomotorischen Centralorgane zurückführen.

\section{Die Wirkung des Amylnitrits anf den Herzsehlag.}

Wie bereits erwähnt, hat Guthrie an sich selbst nach Inhalation sehr geringer Mengen Amylnitrits eine Pulsbeschleunigung beobachtet, welche bis zum Doppelten der normalen Frequenz ging. Für den Menschen ist dies seitdem allseitig bestätigt worden. Beim Hunde fand Wood ${ }^{1}$ ) und nach ihm Amez-Droz ${ }^{2}$ ) und Pick ebenfalls ein sehr erhebliches Ansteigen der Frequenz. In Bezug auf das Kaninchenherz liegen nur von Lauder Brunton und von Eulenburg und Guttmann Angaben vor; diese Autoren leugnen jeden Einfluss des inhalirten Amylnitrits auf die Schlagzahl. Dass auch am Kaninchenherzen eine Steigerung der Pulsfrequenz durch das Amylnitrit hervorgerufen werde, ist von Niemand angegeben. Ebensowenig hat sich irgend ein Autor darüber ausgelassen, woher es kommen könnte, dass in dieser Beziehung das Kaninchenherz sich so ganz anders verhalte, als die Herzen der andern Warmblüter. Ich werde später zeigen, dass auch das Herz des Kaninchens unter der Einwirkung des Amylnitrits constant eine Beschleunigung seiner Action zeigt und daher durch dieses Mittel ganz so wie die Herzen des Menschen und des Hundes beeinflusst wird.

Am Frosche soll, nach Pick, das Amylnitrit bei allen Applicationsweisen keine Beschleunigung des Herzschlages, sondern nur

1) Americ. Journal of the med. sc. (1871) Vol. LXII. p. $39-65$ und ebenda p. 359.

2) l. c. p. 479 (Exp. $\nabla I)$. 
anfänglich eine Kräftigung, später eine Kraft- und Frequenzverminderung der Herzaction verursachen.

Ich habe zunächst an Fröschen, Kaninchen und Menschen die soeben berichteten thatsächlichen Angaben einer neuen Prüfung unterzogen, um eine genügend sichere Unterlage für fernere Untersuchung zu haben.

Bei Fröschen fand auch ich wie Pick keine Zunahme in der Frequenz des Herzschlages, wenn ich sie in eine mit Amylnitritdämpfen erfültte Luft brachte. Ebenso kann ich die Angabe Pick's bestätigen, dass bei längerer Einwirkung und bei grösseren Gaben oder bei subcutaner und interner Anwendung des Mittels die Herzschlagfrequenz langsam abnimmt. Ein Kräftigerwerden der Herzaction habe ich dagegen nicht gesehen. Die Angabe Wood's und Pick's, dass die directe Application von Amylnitrit auf das Froschherz dasselbe schneller erlahmen lasse, habe ich bewährt gefunden. Hinzufügen kann ich, dass die lähmende Wirkung sich viel schneller an dem Herzen entfaltet, wenn dasselbe aus dem Froschkörper entfernt ist. Ich kam auf die Vermuthung, dass diese promptere Wirkung am ausgeschnittenen Froschherzen dadurch herbeigeführt sein möchte, dass dieses während der Diastole eine gewisse Menge Amylnitrit in seine Höhle aufnähme. Zur Prüfung dieser Annahme injicirte ich eine Spur Amylnitrit in das Innere des Herzens eines sonst intacten Frosches und das Herz stand augenblicklich für immer still.

Jedenfalls ist es sicher, dass beim Frosche das Amylnitrit in keiner Dosis und bei keiner Applicationsweise eine Pulsbeschleunigung verursacht.

Beim Kaninchen habe ich, wie bereits bemerkt, im Gegensatze zu den vorhandenen Angaben constant eine Pulsbeschleunigung gefunden, wenn ich die tracheotomirten Thiere bei freier Athmung geringe Mengen Amylnitrits inhaliren liess.

$\mathrm{Zu}$ diesen Versuchen wurde in das Herz des Versuchsthieres eine Acupuncturnadel gestochen, welche an ihrem stumpfen Ende eine kleine Siegellackkugel trug; diese schlug bei jeder Systole gegen ein passend fixirtes Champagnerglas. Der zählende Beobachter zählte nach dem Gehör jedesmal nur von 1 bis 10 und fing dann wieder mit 1 an; jede gezählte 10 wurde in irgend einer Weise markirt (z. B. durch einen Kreidestrich). Es wurden stets Viertelminuten- 
Zählungen gemacht. Auf diese Weise kann man bekanntlich selbst enorm hohe Pulsfrequenzen genau ermitteln.

In den ersten Versuchen liess ich die Kaninchen noch, wie es Eulenburg und Guttmann thaten, die Amylnitritdämpfe durch die Nase einathmen. Hierbei zeigten sich die schon oben näher gewürdigten, von der Trigeminus-Reizung ausgehenden Reflex-Erscheinungen: die Thiere hielten mit der Athmung inne, sie wurden sehr unruhig; ihre heftige Muskelaction trübte die Auffassung der Erscheinungen am Herzen; ausserdem kam es zu reflectorischer Erregung des Vaguscentrums (ebenfalls von der Nasenschleimhaut her) und so zu einer erheblichen Verlangsamung des Herzschlages, welche die specifische Wirkung unseres Mittels verdeckte. Dennoch liess sich schon in diesen Versuchen der specifische, pulsbeschleunigende Einfluss des Amylnitrits erkennen. Ein Kaninchen, welches vor dem Beginne der Inhalation 52 Herzcontractionen in der Viertelminute zeigte, hatte nach der ersten Inhalation derer nur noch 10 (reflectorische Erregung des Herzhemmungssystems von der Nasenschleimhaut aus). Als nach Verlauf von $1-1 \frac{1}{2}$ Minute unter grosser Unruhe des Thieres die Amylnitritwirkung sich an den Ohrgefässen documentirte, wurde der mit dem Mittel getränkte Wattebausch aus der Nähe des Kaninchens entfernt: die Herzaction betrug jetzt 75 in der $1 / 4$ Minute, war also um fast $50 \%$ gegen die Norm gesteigert: Gegen die Beweiskraft dieses Resultates lassen sich zwei Einwände erheben. Erstens könnte das Vaguscentrum, welches vorher durch den Reflex von der Nasenschleimhaut her stark erregt worden war, in einen Zustand von Ermüdung versetzt sein, so dass durch diese die Pulsfrequenzsteigerung erklärt wäre, ohne dass unser Mittel einen specifischen Einfluss auf das Herz gehabt hätte: dieser Einwand ist jedoch nicht stichhaltig; denn wenn man die Nasenschleimhaut durch einen Kohlensäurestrom reizt und auf diesem Wege den gleichen Reflex hervorruft, so folgt auf die Pulsfrequenzverminderung niemals eine erhebliche Frequenzsteigerung. Zweitens aber könnte mir entgegengehalten werden, dass die in meinem Versuche beobachtete Pulsvermehrung nicht von dem Amylnitrit als solchem, sondern von der vorgängigen psychischen und körperlichen Unruhe des Thieres abhängen könnte. Für die Auffassung von einer specifischen Wirkung des Amylnitrits auf das Kaninchenherz sprach der Umstand, dass die Rückkehr der Pulsfrequenz zur Norm 
gleichen Schrittes geschah mit dem Nachlass der Dilatation an den Ohrgefässen.

Den erörterten, sowie einigen andern naheliegenden Einwänden bin ich nun durch Versuche begegnet, in denen ich die Kaninchen tracheotomirte und dann durch eine Canüle frei athmen liess. Zwei bis fünf ohne Beschwerde ausgeführte Inhalationen genügten, um den Herzschlag von circa 50 auf 75 in der Viertelminute steigen zu lassen, während die Ohrgefüsse sich in äusserstem Maasse erweiterten. Diese Erscheinung ist durchaus constant und ich betrachte es daher als erwiesen, dass auch beim Kaninchen die Inhalation geringer Mengen Amylnitrits die Pulsfrequenz erheblich vergrössert 1).

Warum haben nun Lauder Brunton und Eulenburg und Gut t mann die Pulsbeschleunigung nicht gesehen? Zuvörderst haben diese Autoren nicht mitgetheilt, in welcher Weise sie die so überaus frequente Herzaction des Kaninchens gemessen haben; es könnte sehr wohl an der Zählungsmethode gelegen haben, dass jene Erscheinung ihnen entgangen ist. Gegen Eulenburg und Guttmann muss ich ausserdem bei der oben geäusserten Vermuthung stehen bleiben, dass bei der von ihnen geübten Inhalationsmethode überhaupt keine nennenswerthen Mengen Amylnitrits in die Lungen der Versuchsthiere gelangt sind; im anderen Falle hätten sie die Veränderungen in der Herzaction, der Ohrgefässfüllung und der

1) Bei dieser Gelegenheit will ich bemerken, dass die in dieser Weise vorbereiteten und mit Amylnitrit-Inhalationen behandelten Kaninchen ausnahmslos eine Steigerung der Respirationsfrequenz um $30-50 \%$ zeigten, sobald und solange die Ohrgefässe dilatirt waren und die Beschleunigung des Herzschlages bestand. Wenn Eulenburg und Guttmann angeben, sie hätten keine Veränderung der Respiration gesehen, so erledigt sich diese Angabe in derselben Weise, wie ihre analoge Behanptung, dass der Herzschlag ungeändert bleibe, worüber oben das Nöthige alsbald gesagt werdeu wird. Da sie an nicht tracheotomirten Kaninchen arbeiteten, so haben sic vermuthlich die Unregelmässigkeiten der Respiration, welche während und nach der Inhalation auftraten, auf Rechnung des Umstandes gesetzt, dass die Nasenschleimhaut durch die Aetherdämpfe gereizt werde. Meine Versuche, frei von dieser Fehlerquelle, scheinen mir die specifische Respirationsvermehrung zu beweisen. Der Angriffspunkt für diese Athmungsbeschleunigung ist übrigens weder in den pulmonalen Ausbreitungen der Vagi, noch im Grosshirn zu suchen, denn die Beschleunigung trat nach Amylnitrit-Inhatation in gleicher Weise auch an Kaninchen auf, denen ich die Vagi durchschnitten und die Grosshirnhemisphären entfernt hatte. 
Respiration zu gleicher Zeit gewiss nicht constant übersehen. La u der Brunton's abweichendes Resultat möchte, abgesehen von der Zählungsmethode, wohl noch darin seine Erklärung finden, dass er, wie ich bereits oben ausführte, seinen Kaninchen viel zu grosse Mengen Amylnitrits einblies. In der That kann man nämlich durch Inhalation so grosser Mengen Amylnitrits, dass allgemeine Krämpfe auftreten (und Lauder-Brunton gibt ja selbst an, stets Krämpfe beobachtet zu haben), nach kurz vorübergehender Steigerung der Pulsfrequenz eine Abnahme derselben constatiren, die sogar bis weit unter die Norm sich erstrecken kann.

Beim Menschen habe ich, wie meine Vorgänger, nach Amylnitrit-Inhalation eine Beschleunigung des Pulses bis zu 144 in der Minute und darüber gesehen.

Fassen wir zusammen, was über den Einfluss gesagt ist, den unser Mittel auf den Herzschlag ausübt, so ergibt sich, dass dasselbe bei Inhalation relativ kleiner Mengen die Zahl der Herzsystolen am Kaltblüter nicht, am Säugethiere und Menschen sehr bedeutend vermehrt, während lang einwirkende, grosse Dosen dieselbe nach und nach vermindert.

Welcher von den bekannten Factoren, die den Herzschlag zu beeinflussen vermögen, die beim Menschen und Säugethiere beobachtete Zunahme der Pulsfrequenz verursacht, ist eine Frage, welche merkwürdigerweise von den bisherigen Untersuchern experimentell nicht in Angriff genommen, geschweige denn entschieden worden ist.

Richards on nimmt ohne experimentelle Unterlage an; dass das Amylnitrit erregend auf das Herz wirke.

Wood 1) glaubt die am Menschen beobachtete Steigerung der Pulsfrequenz davon abhängig, dass das Amylnitrit dem Blute die Fähigkeit nimmt, arterialisirt $\mathrm{zu}$ werden; hierdurch soll eine gewissermaassen innere Erstickung entstehen und diese soll wie jede andere Erstickung zur Herzschlagsbeschleunigung führen. Dies ist eine unerwiesene Annahme. Die Beobachtungen bei $\Lambda$ thmungssuspension an curaresirten Thieren sprechen schlagend gegen dieselbe: Wenn ferner ein Mensch willkürlich den Athem so lange anhält, bis er, überwunden von dem Bedürfnisse, mehrere tiefe schnell auf einander folgende Athemzïge ausführen muss, so findet gegen das

1) 1. c. p. 359 und 360 . 
Ende der freiwilligen Pause durchaus keine wesentliche Beschleunigung des Herzschlages statt, obwohl doch das Blut in so hohem Grade sauerstoffarm und kohlensäurereich geworden ist, dass es eine heftige Athemnoth hervorbringt. Nach Inhalation geringer Mengen Amylnitrits besteht beim Menschen eine nur sehr geringe Vergrösserung des Athmungsbedürfnisses und dennoch ist die Pulszahl verdoppelt. Die Sauerstoffarmuth kann also nicht die Ursache der Pulsbeschleunigung sein. Weiter unten werde ich überdies zeigen, dass das Amylnitrit unter bestimmten Versuchsbedingungen keine Wirkung auf das Herz ausübt, obwohl das mit demselben imprägnirte Blut zu dem musculomotorischen Herznervensysteme gelangt, was selbstverständlich nicht möglich wäre, wenn Wood's Annahme das Richtige getroffen hätte. Im Uebrigen beweist die schöne rothe Farbe, welche man an gesunden Menschen nach Inhalation geringer Mengen Amylnitrits beobachtet, dass durch solche geringe Mengen das Blut nicht in der Weise verändert wird, wie Wood annimmt. Vergiftet man Thiere (Frösche, Kaninchen) mit sehr grossen, tödlichen Mengen Amylnitrits, so nimmt das Arterienblut allerdings die von Wood beschriebene braune Farbe an. Jedenfalls ist aber Wood's Erklärung der Pulsbeschleunigung schon an sich unrichtig.

Die übrigen Autoren äussern sich gar nicht über die inneren Ursachen der Herzschlagsvermehrung. Die Bemerkung AmezDroz's 1): "Quant à l'accélération de l'action du coeur, elle dépend de la dilatation des capillaires, " ist wohl kaum als ein Erklärungsversuch in Betracht zu ziehen.

Wenn wir uns anderseits in der vor uns liegenden Literatur nach Thatsachen umschauen, die für eine Erklärung der Pulsfrequenzzunahme verwerthet werden könnten, so gewinnen wir auch hier eine sehr spärliche Erndte.

Aus den Protocollen Lauder Brunton's kann man ersehen, dass seine Amylnitrit-Einblasungen an den Kaninchen nach Halsmarkdurchschneidung keine Steigerung der Pulszahl verursachten. Da er aber auch am intacten Kaninchen die Frequenzsteigerung nicht $\mathrm{zu}$ Gesicht bekommen hat, so ist auch diese letztere Angabe nicht sicher zu verwerthen; im andern Falle hätte man aus ihr schliessen können, dass das Amylnitrit erregend auf die beschleunigenden Herznerven wirke, da die Beschleunigung ausblieb, als die

1) 1. c. p. 503 . 
beschleunigenden Herznerven mittels Halsmarkdurchschneidung ausgeschaltet waren.

In demjenigen Abschnitte seiner Arbeit ${ }^{1}$ ), in welchem Wood über den etwaigen Einfluss spricht, welchen das Herz auf das Zustandekommen der Blutdrucksverminderung haben könnte, ist ein Experiment (Nro. 20) enthalten, welches einen Beitrag zur Lösung der uns beschäftigenden Frage zu liefern scheint. Dort sind einem Hunde beide Vago-Sympathici durchschnitten; in die eine Carotis ist ein Manometer eingeführt; die Pulszahl wird mit 180 in der Minute angegeben (in welcher Weise der Puls gezählt wurde, ist nicht gesagt; aus vorhergehenden Bemerkungen könnte man jedoch schliessen, dass die Herzelevationen des Quecksilbers im Manometerrohre gezählt wurden). Dem Hunde wird etwas Amylnitrit in die Bauchhöhle gespritzt; nach 15 Minuten zeigen sich 204 Pulse in der Minute. Es ist hier also nach Application des Amylnitrits bei durchschnittenen Vagis eine Steigerung der Pulsfrequenz um 13,3\% eingetreten. Wäre dieser Versuch vorwurfsfrei, so würde man aus ihm schliessen können, dass die Herzbeschleunigung am intacten Thiere nicht ausschliesslich und allein auf einer Aufhebung des Vagustonus beruhen könne. Anderseits müsste man bei der geringen Vermehrung in jenem Versuche $(13,3 \%)$ und in Anbetracht dessen, dass beim Menschen und unversehrten Hunde durch Amylnitrit eine Frequenzsteigerung bis zu $100 \%$ verursacht wird; doch wohl darauf denken, dass der Fortfall des Vagustonus einen wesentlichen Antheil an der Pulsbeschleunigung des intacten Thieres haben möchte. Indessen verlohnt es sich nicht, auf diese Betrachtungen näher einzugehen, denn das Experiment ist nicht verwerthbar. Ganz abgesehen von der Unzweckmässigkeit der Applicationsweise (Einspritzung in die Bauchhöhle), die es ungewiss lassen muss, ob die injicirte Flussigkeit in die Peritonealhöhle oder in das Darmlumen oder sonst wohin gelangt ist; ganz abgesehen ferner davon, dass die Injection in die Peritonealhöhle wegen der durch sie veranlassten Schmerzen die Herzaction beschleunigen könnte, ohne dass ein specifischer Einfluss auf dieselbe vorläge, ist die ganze Anordnung des Wood'schen Versuches nicht geeignet, ein beweisendes Resultat in Beziehung auf unsere Frage $\mathrm{zu}$ liefern. Der Hund ist weder curaresirt, noch narcotisirt. Da der Blutdruck manometrisch gemessen wird, so

1) 1. c. p. $51 \mathrm{fif}$. 
muss der Hund aufgebunden sein. Die Schmerzen, die Aufregung des psychisch so hoch stehenden Versuchsthieres, die Muskelanstrengung, die ein nicht curaresirter und nicht narcotisirter Hund in solcher Situation stets macht, sind mehr als genügend, um die von $\mathrm{W}$ o od gefundene, verhältnissmässig doch nur geringe Pulsfrequenzsteigerung zu erklären. Uebrigens ist auch das Ablesen so hoher Pulszahlen aus den Quecksilberschwankungen durchaus unsicher, wenn das Versuchsthier nicht absolut ruhig liegt. Am meisten muss aber der Wood'sche Versuch dadurch an Gewicht verlieren, dass er nur e in Mal gemacht wurde, und dass auch in diesem einen Versuche nur eine einzige Einzelbeobachtung vorliegt. Wäre in jenem Versuche die Herzfrequenz nach Elimination des Amylnitrits wieder auf die ursprüngliche Höhe gegangen und wäre sie stets bei jeder neuen Darreichung des Mittels wieder gestiegen, so hätte diese Beobachtung für uns trotz aller gerügten Versuchsmängel doch Werth gehabt. Wie die Dinge aber liegen, muss jenem von W ood übrigens gar nicht zur Entscheidung der uns hier bèschäftigenden Frage angestellten Versuche jede Beweiskraft für uusern Zweck abgesprochen werden.

Anderweitige Thatsachen, welche über die näheren Ursachen der durch das Amylnitrit herbeigeführten Pulsbeschleunigung uns einiges Licht geben könnten, liegen meines Wissens nicht vor.

Indessen lässt der Unterschied in der Reaction, welchen dem Amylnitrit gegenüber das Säugethier- und das Froschherz zeigen, doch schon eine Vermuthung darüber aufkommen, welches der Entstehungsmodus der beim Säugethiere nach Amylnitrit-Inhalation auftretenden Pulsbeschleunigung sein möchte. Bei der sonst so durchgehenden Analogie, nach welcher sich die Herzen der beiden Wirbelthierreihen (Warm- und Kaltblüter) auf toxische Substanzen reagiren, ist es im vorliegenden Falle nicht unwahrscheinlich, dass beim Säugethiere gerade derjenige Factor durch das Amylnitrit beeinflusst werde, welcher ausschliesslich den wesentlichsten Unterschied zwischen der Herzinnervation des Warmblüters und derjenigen des Frosches ausmacht - und dies ist der Vagustonus, der, beim Warmblüter von hervorragender Wirksamkeit, beim Frosche so gut wie nicht vorhanden ist.

Eine andere Thatsache ist durchaus geeignet, diese Vermuthung $\mathrm{zu}$ stützen. Aus meinen Versuchen war wie erwähnt, hervorgegangen, dass beim Kaninchen die Pulsfrequenz nach Inhalation 
Ueber den Einfluss des Amylnitrits auf Gefässtonus und Herzschlag. 489

geringer Mengen Amylnitrits sehr bald die Zahl 75 (in der Viertelminute) erreichte und nie erheblich überschritt. Nun macht aber ein Kaninchenherz, wenn beide Vagi durchschnitten sind, auch etwa 75 Systolen in der Viertelminute, und es lag nahe, im ersten Falle die gleiche Ursache wie im zweiten anzunehmen, d. i. die Elimination des Vaguscentrums.

Aus den Versuchen, die ich unternommen habe, um diese Annahme auf die Probe zu stellen, ist nun in der That hervorgegangen, dass die Ursache der beobachteten Beschleunigung des Herzschlages ausschliesslich in der Aufhebung des Vagustonus gelegen ist.

Zunächst durchschnitt ich an einem (tracheotomirten) Kaninchen beide Vagi. Nachdem seine Herzaction, die nach der oben beschriebenen Methode gemessen wurde. mehrere Minuten hindurch beständig 76 in der Viertelminute gezeigt hatte, liess ich das Thier Amylnitrit inhaliren. Dass das Mittel seine Wirkung entfaltete, bewies die Blutüberfüllung der Ohrgefässe. Die Pulsfrequenz stieg jedoch nicht mehr an. Auch die Darreichung grösserer Mengen Amylnitrits vermehrte die Pulsfrequenz nicht; im Gegentheil nahm schliesslich, nachdem Krämpfe eingetreten waren, die Frequenz etwas ab.

Diese Beobachtung beweist, dass, nach Ausschaltung des Vaguscentrums, die übrigen Factoren, welche die Herzinnervation beherrschen, vom Amylnitrit nicht beeinflusst werden. Daher ist die Vermehrung der Herzschläge, welche bei erhaltenen Vagis nach Amylnitrit beobachtet wird, auf Rechnung der Lähmung des Vaguscentrums zu setzen.

Es blieb jedoch hierbei noch ein Einwand übrig, der dieser Schlussfolgerung von übermässig skeptischer Seite hätte gemacht werden können. Man hätte vielleicht daran denken können, dass der Herzmuskel' des Kaninchens nicht mehr als 76 Systolen in der Viertelminute zu leisten im Stande sei, und dass alsdann ein ferneres Wachsen der Zahl der nervösen Impulse keinen Einfluss mehr auf die Schlagzahl auszuüben vermöge. In diesem Falle wäre aus dem Mangel einer Frequenzzunahme nicht auf das Fehlen neuer nervöser Antriebe zu schliessen. Auch diesem durch anderweitige Thatsachen wohl kaum gestützten Einwande gegenüber entschied das Experiment in dem Sinne, dass geringe Mengen Amylnitrits den Tonus des Vaguscentrums aufheben und die andern das Herz beherrschenden Innervationen ungeändert lassen.

Der Versuch wurde in folgender Weise angestellt. 
Einein (tracheotomirten) Kaninchen, dessen Herz 54 Systolen in der Viertelminute ausführt, werden beide Vagi durchschnitten. Die Herzaction beträgt jetzt 76. Das peripherische Stück des rechten Vagus wird über ein Electrodenpaar gebrückt, welches mit der secundären Rolle eines Inductionsschlittens in Verbindung steht. Von unwirksamen Stromstärken beginnend, verstärke ich nach und nach die Inductionsschläge, bis das Herz nur noch 58 Schläge in der Viertelminute macht. Jetzt hat also das Herz des Kaninchens die normale Schlagzahl, während es vom Vagustonus und dessen Schwankungen völlig unabhängig, dagegen allen andern auf dasselbe einwirkenden Factoren in normaler Weise zügängig ist. Die jetzt veranlasste Inhalation von Amylnitritdämpfen bewirkt kein e A enderung der Pulsfrequenz, auch nicht, wenn ich das Mittel bis zum Eintritte allgemeiner Krämpfe darreiche, während gleichzeitig die Blutüberfüllung der Ohren das Statthaben der Amylnitritwirkung beweist.

Es wird also nach Elimination des Vagascentrums auch bei (niedriger) normaler Frequenz des Herzschlages keiner der übrigen Herzinnervatoren durch solche Gaben Amylnitrits beeinflusst, die am unverletzten Thiere eine Beschleunigung des Herzschlages verursachen, und daher ist letztere einzig und allein auf eine Aufhebung resp. Verminderung des Tonus im Vaguscentrum zurückzuführen.

Hiermit wären die Fragen, die wir uns im Beginne unserer Untersuchung gestellt hatten, genügend beantwortet.

Zum Schlusse sei es mir gestattet, auf die Analogie einzugehen, welche zwischen der Wirkung des Amylnitrits und derjenigen gewisser psychischer Vorgänge, z. B. der Schüchternheit und des Beschämtseins besteht. Darwin hat, wie erwähnt, zuerst auf die Aehnlichkeit aufmerksam gemacht, die das Rothwerden nach Amylnitrit mit dem Erröthen vor Scham hat. Mit dieser Aehnlichkeit ist jedoch die vorhandene Analogie nicht erschöpft. Beiden Zuständen ist auch noch das Herzklopfen und die beschleunigte Athmung gemein. Es ist nun äusserst interessant zu sehen, wie der gleiche Complex von Erscheinungen, welcher durch einen ausschliesslich dem Menschen eigenthümlichen, höheren psychischen Vorgang hervorgerufen wird, auch experimentell durch Spuren eines Aethers an Mensch und Säugethi er erzeugt werden kann. Es ist wohl nicht zu gewagt, wenn wir annehmen, dass beide Einflüsse, das Amyl- 
Ueber den Einfluss des Amylnitrits auf Gefässtonus und Herzschlag. 491

nitrit und der psychische Vorgang an gleichen Puncten des Nervensystems angreifen, um die gleichen Erscheinungen hervorzubringen. Danach ist es durchaus wahrscheinlich, dass das Herzklopfen der Schüchteruheit ebenso wie dasjenige der Amylnitrit-Intoxication auf einer Aufhebung des Vagustonus beruht. Es scheint ferner im Hirn eine ganz besonders enge Verknüpfung zwischen dem Vaguscentrum und demjenigen Abschnitte des vasomotorischen Centralapparates zu bestehen, welcher die Gefässe des Kopfes beherrscht, so dass die gleiche Ursache, welche den Tonus des ersteren aufhebt, auch mit Leichtigkeit die Thätigkeit des letzteren sperrt. Die vermehrte Erregung des respiratorischen Apparates ist bei den besprochenen Vorgängen vermuthlich keine directe; denn es ist unwahrscheinlich, dass dieselbe Substanz oder derselbe Vorgang das eine Centrum lähme und das andere errege; vielmehr will es uns glaublicher erscheinen, dass die in Folge der Gefässlähmung und der Herzschlagbeschleunigung so wesentlich veränderte Blutcirculation die Ursache der gesteigerten Athmungsthätigkeit ist.

Die herangezogene Analogie zwischen der Wirkung des Amylnitrits und derjenigen des Beschämtseins beseitigt die Vorstellung, als ob die Fähigkeit, unter Herzklopfen und beschleunigter Athmung zu erröthen, ein Privilegium des Menschen wäre; sie zeigt uns, dass die andern Säugethiere für gewöhnlich nur desshalb nicht erröthen, weil von ihrer minder entwickelten Psyche kein Antrieb zur Abspielung jenes interessanten Vorganges gegeben wird; schafft man den Antrieb durch Darreichung von Amylnitrit, so zeigt es sich, dass bei den Säugethieren derselbe nervöse Mechanismus vorhanden ist, wie bei uns. Diese Betrachtungsweise scheint mir das Verständniss dafür zu erleichtern, wie sich bei den Menschen im Verlaufe ihrer Veredlung jener eigenthümliche Verräther ihrer innern Empfindungen mit allen seinen Begleiterscheinungen hat herausbilden können. 